\title{
Comparative genomics of swine leukocyte antigen class I of Nigerian and Kenyan pigs
}

${ }^{1}$ Oluwole, O. O., ${ }^{2}$ Bayene, D., ${ }^{3}$ Okoth, E., ${ }^{3}$ Roger, P. and ${ }^{4}$ Omitogun, G. O.

${ }^{I}$ Obafemi Awolowo University, Institute of Agricultural Research and Training, P.M.B. 5029, Moor Plantation, Ibadan, Nigeria; ${ }^{2}$ Addis Ababa Ethiopia. ${ }^{3}$ Biosciences for Central Africa, International Livestock Research Institute, Nairobi Kenya.

${ }^{4}$ Obafemi Awolowo University, Department of Animal Sciences, Ile-Ife.

"Corresponding author: oluwafunmike@yahoo.co.uk; +234(0)8053570807

\section{Abstract}

The classical genes are a member of immunoglobulin gene family that is involved in the presentation of antigen peptides. They are located in every nucleated cell surface, except in neurons and trophoblasts. They are synthesized in infected cell (viral infection), binding to viral proteins and giving rise to Swine Leucocyte Antigen (SLA) I- antigen complex. In the study, the SLA class I gene of Nigerian pigs with pigs from Kenya along with archived sequences from Asia and American pigs available on Genbank to gain a better understanding of matrilineal origin of Nigerian and Kenyan pigs. Gain insight into demographic distribution, their domestication and adaptation were compared. The deoxyribonucleic acid (DNA) was extracted from 90 pigs (60 Nigerian pigs (NP) and 30 pigs from Kenya) using Invitrogen Kit, quantified by using Nanodrop, and amplified by using Polymerase Chain Reaction. The expected bands were purified and sequenced for SLA class I. The sequences were aligned and phylogenetic tree was analyzed by using MEGA 6. BLAST (Basic Local Alignment Search Tools) was conducted in NCBI (National Center for Biotechnology Information). The sequences of Nigerian pigs and Kenya pigs were compared with published pig data in GenBank. The Neighboring Joint (NJ) tree constructed with sequences from Nigeria, Kenya and other pigs from GenBank revealed that some of the NP clustered together with Asian pigs at the middle of the tree while some clustered just above the Kenyan below the tree and the outgroup homosapien stood out. The result from the calculation of total number of segregating sites gave the negative Tajima's D value (-0.181) while the non-significant difference $(p>0.05)$ obtained from the result showed that the null hypothesis was not rejected. In conclusion, there is genetic difference between the Nigerian and Kenyan pigs based on the phylogeny tree constructed. There is an excess of low frequency polymorphisms relative to population size. This may be as a result of evolutionary forces during course of adaptation in tropical countries in Africa.

Keywords: Nigerian pigs, Kenya pigs, Swine Leukocyte Antigen class I

\section{Introduction}

Over 90 breeds of pig were recognized in the world and 230 varieties were estimated (Ikanni and Dafwang, 1995) Domestic pigs can be grouped into exotic, indigenous and their crossbreeds. The common breeds found in Nigeria are NIP or Local pigs and the improved pigs from crosses between the NIP and Exotic breeds especially Large White and Landrace. Swine Leucocyte Antigen Genes Class I can be classified into classical and non-classical genes. The classical genes are a member of immunoglobulin gene family that is involved in the presentation of antigen peptides. They are located in every nucleated cell surface, except in neurons and trophoblasts. While they are synthesized in infected cell (viral infection), binding to viral proteins and hence giving rise to an SLA I- antigen complex. This complex will be recognized by certain $\mathrm{T}$ 


\section{Comparative genomics of swine leukocyte antigen class I of Nigerian and Kenyan pigs}

lymphocytes, CD8+ cytotoxic T cells that kill the infected cells. Antigen presentation in infected cells to CD8+ is one of the main functions of SLA I (Vaiman et al., 1970). Its structure consists of two chains: a heavy chain, of $45 \mathrm{KDa}$ (KiloDalton), with a great polymorphism. It is codified by SLA genes and known as the A chain. The other chain is a light chain, which is non-covalently associated with the swine 32 microglobulin, a polypeptide of $11.7 \mathrm{kDa}$ called B. The heavy chain is a transmembrane molecule, encoded within the SLA complex, which expresses all the polymorphisms observed. The objective of this study was to compare SLA class I gene of Nigerian pigs with pigs from Kenya along with archived sequences from Asia and American pigs available on Genbank to gain a better understanding of matrilineal origin of Nigerian and Kenyan pigs, gain insight into demographic distribution and their domestication and adaptation.

\section{Methodology}

Genomic DNA was extracted from blood spots (FTA cards) of 60 pigs from Nigeria, using kit (Invitrogen) at the Biosciences for east Africa-International Livestock Research Institute hub (BecA-Ilri hub, Nairobi, Kenya. The DNA concentration and quality were checked using Nanodrop. The primer pair sequence for SLA class I forward and Reverse primers were: T7$\begin{array}{llllll}A & 1 & p & h & a & -\end{array}$ TAATACGACTCACTATAGGGACCGC CGTGTCCCGGC(C/T)CGAC and Reverse: Sp6-Alpha-ATTTAGGTGACACTATAGCCTCGCTCTGGTTGT AGTAGC primers. The PCR reaction volume was performed in $25 \mu \mathrm{l}$ containing of $100 \mathrm{ng}$ genomic DNA, $2.5 \mu \mathrm{L}$ of $10 \times \mathrm{PCR}$ buffer. The thermal cycling conditions were as followed; initial temperature at $94^{\circ} \mathrm{C}$ for 5 minutes, followed by 35 cycles at $94^{\circ} \mathrm{C}$ for 30 minutes, step up annealing temperatures at $59-60^{\circ} \mathrm{C}$ for 45 minutes, extension at $72^{\circ} \mathrm{C}$ for 45 seconds, with a final extension of 5 minutes at $72^{\circ} \mathrm{C}$. PCR products were electrophoresed in 1\% low-melting point agarose gels (Promega) in 1X TAE. The products of expected size were excised and purified with extraction kit (Thermoscientific) and sequenced at Macrogen (USA). The nucleotide analysis was done by using CLC workbench version 7.6.4. Blast was conducted in NCBI (National Center for Biotechnology Information) using Blast $\mathrm{n}$ option to get the similar or homologous sequences from other breed of pigs from GenBank (http \\blast.ncbi.nlm.nih.gov/Blast/). Alignment of sequences was carried out by using CLC workbench version 7.6.4 and Clustal W algorithms in Bioedit sequence alignment editor software. The phylogenetic tree was constructed by using MEGA 6. The confidence of branching order was estimated by the use of the bootstrap analysis using 1000 replicates. Haplotype diversity, nucleotide diversity, number of segregating site and Tajima's D were estimated by using DnaSP version 5 .

\section{Results and discussion}

The results obtained from the Neighboring Joint (NJ) tree constructed by using MEGA 6 (Talima et al., 2013) with sequences from Nigeria, Kenya and other pigs from GenBank revealed that some of the NP clustered together with Asian pigs at the middle of the tree while some clustered just above the Kenyan below the three and the outgroup homosapien stood out as shown in Figure 1. Great differences were observed between the pigs from different countries and sources. The clustering of NIP MHC class 1 gene with Asian pigs was in line with the findings of Oluwole et al. (2016) where the NP SLA class II gene at the exon two and three regions clustered with that of Asian pigs. Macromolecules, as described by 


\section{Oluwole, Bayene, Okoth, Roger and Omitogun}

Brinkman and Leipe (2001) data for phylogenetic or cladistics character is the most accepted form than that of morphological and other organismal character. This result show that sequences that clustered together are closely related and there was a high degree of average relatedness among genotypes within the same cluster. Moreover, there is possibility that genotypes within the same cluster had similar genetic background. The NP consisting Nigerian Indigenous pigs (NP 13 ), crossbreds (NP 4-6) and exotic pigs (NP 7-8). The NP seven and eight clustered between the Asian and America pigs. Haplotype, haplotype diversity and nucleotide diversity were 19,0.98 and 0.36, respectively (Table 1). The low haplotype results in evolutionary potential of livestock to rapidly adapt to change in environmental condition. According to Frankham et al. (2002), the nucleotide diversity is affected by several factors which includes selection, mutation rates, mating system, effective population size, and demography, gene flow between populations, introgression from hybridization and historical effects on these factors (e.g., population bottlenecks).

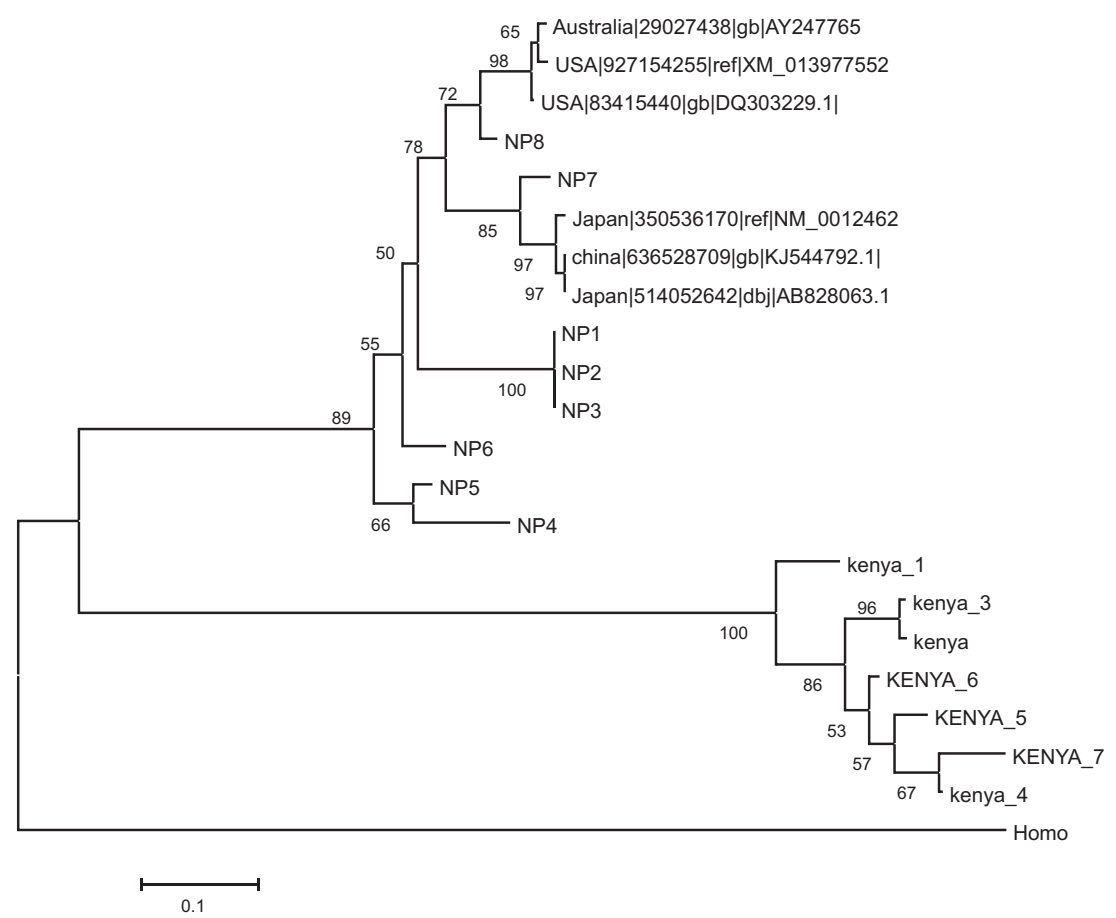

Figure 1: Phylogeny of Nigerian pigs, Kenya pigs and other pigs from genBank NP-Nigerian pigs, Homo-homosapien

Table 1: Population Statistical and Neutrality Test

\begin{tabular}{|c|c|c|c|c|c|c|c|}
\hline \multirow{2}{*}{\multicolumn{3}{|c|}{ Population Statistics }} & \multicolumn{5}{|c|}{ Neutrality Test } \\
\hline & & $\mathrm{Hd}$ & $\mathrm{S}$ & $\mathrm{Pi}$ & 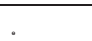 & Tajima'sD & $\mathrm{NS}$ \\
\hline 23 & 19 & 0.98 & 226 & 0.36 & 0.382 & -0.181 & Not significant \\
\hline
\end{tabular}




\section{Comparative genomics of swine leukocyte antigen class I of Nigerian and Kenyan pigs}

Tajima's D statistics test result from the calculation of total number of segregating sites gave a negative value $(-0.181)$ (Table 1). This negative Tajima's D result signifies an excess of low frequency polymorphisms relative to expectation, indicating population size expansion (e.g., after a bottleneck or a selective sweep) and/or purifying selection. Also, the nonsignificant difference $(p>0.05)$ obtained from the result shows that the null hypothesis was not rejected. This indicates that the evolution of SLA class I gene is caused by random drift of selectively neutral mutants. This result was in line with Yang et. al. (2013) and Wlasiki et al. (2009) where the values obtained were nonsignificant for Toll like receptor.

\section{Conclusion}

The study showed that there was genetic difference between the Nigerian and Kenyan pigs based on the phylogeny tree constructed. There was an excess of low frequency polymorphisms relative to population size. This may be as a result of evolutionary forces during course of adaptation.

\section{Acknowledgement}

"The laboratory aspects of this project was partly/fully supported by the BecA-ILRI Hub through the Africa Biosciences Challenge Fund (ABCF) program and Africa women in Agriculture and development (AWARD). The ABCF Program is funded by the Australian Department for Foreign Affairs and Trade (DFAT) through the BecA-CSIRO partnership; the Syngenta Foundation for Sustainable Agriculture (SFSA); the Bill \& Melinda Gates Foundation (BMGF); the UK Department for International Development (DFID) and; the Swedish International Development Cooperation Agency (SIDA)."

\section{References}

Brinkman, F. S. L. and Leipe, D. D. 2001. Phylogenetic Analysis. In: Bioinformatics: A Practical Guide to the Analysis of Gene and Protein. Baxevanis, A. D. and B. F. F.Ouellette (Eds.). John Willey \& Sons. pp. 323-358.

Frankham R., J. D. Ballou, D. A. Briscoe. 2002. Introduction to Conservation G e n e ti c s; C a m bridge University Press: Cambridge, UK.

Ikani, I. E. and Dafwang, I. I. 1995. Pig Production technology for piggery farmers, Extension bulletin No 25. Livestock series No 1 NAERLS, A.B.U Zaria.

Oluwole, O., Bayene D., Okoth, E., Roger P., Omitogun G. O. 2016. Comparative Genomics of Swine Leukocyte Antigen Class I of Nigerian and Kenyan Pigs. In Proceedings of the biennial Conference of the Ghana Society of Animal Production.

Tajima, F. 1989. Statistical-method for testing the neutral mutation hy pothesis by D N A polymorphism. Ge netics.; Pp.123:585-595.

Vaiman, M., Renard, C., Lafage, P., Ameteau, J. and Nizza, P. 1970. Evidence for a histocompatibility system in swine (SLA). Transplantation 10:155-164.

Wlasiuk, G., Khan, S., Switzer, W. M. and Nachman, M. W. 2009. A history of recurrent positive selection at the toll-like receptor 5 in primates. Mol Biol Evol.; 26:937-949.

Yang, X., Haitao L., Qingzhi, G. and Liu, D. 2013. Genetic diversity of Tolllike receptor 5 among $\mathrm{p}$ i $\mathrm{g}$ populations. Genet Mol Biol. 36(1):37-42.

Received: $18^{\text {th }}$ March, 2019

Accepted: $26^{\text {th }}$ July, 2019 